\title{
59
}

\section{ON SOME OBJECTIONS TO MIMICRY THEORY; STATISTICAL AND GENETIC}

By B. A. FISHER, Sc.D.

(Rothamsted Experiment Station, Fellow of Gonville and Caius College.)

(Communicated by Prof. E. B. POULTON, F.R.S.)

[Read October 5th, 1927.]

\section{INTRODUCTORY.}

THE great statistical interest of all applications of selection theory, of which mimicry is certainly one of the most extensive and detailed, makes the validity of the latter a matter of importance even beyond the limits of the biological sciences. Moreover, it appears to be probable that disputed points which have arisen in discussions of mimicry, should, in so far as they are of a purely statistical nature, be capable now of a definite and final decision. The importance of inheritance in selection theory has led to a study of the statistical effects of Mendelism, with the result that the genetical arguments are in nearly the same position as the purely statistical. It is now becoming increasingly widely understood that the bearing of genetical discoveries, and in particular of the Mendelian scheme of inheritance, upon evolutionary theory is quite other than that which the pioneers of Mendelism originally took it to be. These were already, at the time of the rediscovery of Mendel's work, in the full current of that movement of evolutionary thought, which in the nineties of the last century, had set in in favour of discontinuous origin for specific forms. It was natural enough, therefore, that the discontinuous elements in Mendelism should, without sufficiently critical scrutiny, have been interpreted as affording decisive evidence in favour of this view. No attempt will here be made to give an extended account of the general bearing of Mendelism upon evolutionary theory; it should, however, be borne in mind that the reinterpretation of the significance of Mendelism in cases of mimicry is but part of a more general recovery of genetical opinion from positions adopted at a somewhat immature stage of the development of that science.

It seems to the author imperative, in undertaking such a task, the value of which depends solely upon its logical cogency, to insist upon the fact that, in criticising biological conclusions, the criticism is solely aimed at their supposed relation to the observations upon which they are based, and is in no way opposed to, or confirmatory of, these observations. Mathematical symbolism will be avoided; nevertheless the work will be mathematical in the sense that for the sake of certainty and precision upon the points discussed, innumerable side issues, some possibly of importance, will be totally disregarded, and the biological facts utilised will be reduced to a mere abstract of their real complexity.

\section{SUPPOSED STATISTICAL LIMITATIONS OF MULLERIAN MIMICRY.}

Certain a priori statistical limitations are supposed to inhere in the theory of Millerian mimicry; these have been most lucidly developed by G. A. K. Marshall (1). Marshall suggests that, for arithmetical reasons, of two equally unpalatable species inhabiting the same region, the less numerous will tend to resemble the more numerous, while the more numerous will not reciprocate 
this tendency. (Marshall does not suggest that the more numerous will tend to decrease the resemblance.) The general purport of his paper is to emphasise the Batesian as opposed to the Müllerian factor in mimicry, and that principally for biological reasons, with which only a professed entomologist should deal. The question, however, as to whether there is or is not such a statistical limitation as Marshall claims to the scope of the Millerian factor, is of the type to which I suggest mathematical reasoning may profitably be applied.

When illustrated by hypothetical type cases nothing is clearer than the distinction between the Batesian and the Millerian factors; if, however, we ask exactly where the line is to be drawn, it is not too easy to give an answer in terms directly referable to the actual bionomic situation. It is admitted that between two equally unpalatable species only the Müllerian relation is possible, and between a species, which would never be unwelcome (if such exist), and one which would never be attacked, any mimicry must be Batesian; but these are extreme possibilities and Marshall insists, as I hope to show, rightly, that the existence of intermediate degrees of palatability gives increased scope to the Batesian factor. I shall also suggest that in cases involving such intermediate degrees, the Millerian factor cannot properly be excluded, and that the distinction between the two agencies cannot be drawn offhand without further consideration.

If we imagine three species, occupying the same region, of which A is highly unpalatable, $\mathrm{B}$ less so, while $\mathrm{C}$ is free from objectionable qualities, then all possible situations may be exhaustively classified as follows (I, II, III, IV), in which, however, the alternative reasons given for the occurrence of any situation $(a, b, c)$, are by no means exhaustive, but might conceivably be much elaborated by detailed biological observations.

I. A, B and C all liable to attack.

II. B and C liable to attack, but not $\mathrm{A}$.

(a) Wise and experienced birds, knowing full well the flavours of each species, are hungry enough to attack $B$, but not hungry enough to attack A.

(b) Birds have attacked A and found it to be unpalatable, but have not yet had sufficiently impressed upon their minds the unpalatable qualities of $\mathrm{B}$.

III. A and C liable to attack, but not B.

(c) Birds have attacked B and found it to be unpalatable, but have not yet had sufficiently impressed upon their minds the unpalatable qualities of $\mathrm{A}$.

IV. C only liable to attack.

In situation I, mimetic resemblance is without effect, while in situation IV, $\mathrm{C}$ would gain by being mistaken for $\mathrm{A}$ or $\mathrm{B}$, and $\mathrm{A}$ or $\mathrm{B}$ would lose by being mistaken for $\mathrm{C}$. $\mathrm{C}$ might, therefore, if the former effect were to exceed the latter, become a Batesian mimic of A or B, while, on the contrary, A and B would gain by emphasising their distinctive colouring, if so they could diminish the danger of confusion with C. Similarly, in situation II, A loses by being mistaken for $\mathrm{B}$, and $\mathrm{B}$ gains by being mistaken for $\mathrm{A}$; while in situation III the reverse is the case; both $\mathrm{A}$ and $\mathrm{B}$ will seem, therefore, to be acted upon by opposing tendencies, one tending towards similarity, and the other towards dissimilarity. It is only when the possible situations are analysed into their suggested causes that it is possible to indicate the resultant effect.

For this purpose we distinguish the "Batesian situation" II (a), from the "Müllerian situations" II (b) and III (c), recognising that this classification need not be exhaustive. It is then seen that Batesian situations are to be distinguished by (i) depending upon differences of unpalatability and (ii) producing a "Batesian tendency" for B to approach A and for A to recede from $\mathrm{B}$, while 
the Millerian situations (b) and (c) do not depend on any difference of unpalatability, but are taken to occur whenever both species are, on occasion, deemed inferior to some alternative food. It is not, however, obvious from the above analysis that the net effect of (6) and (c) will be to cause a mutual though possibly unequal approach between the two species; such a mutual tendency to approach will be called a 'Müllerian tendency." It is Marshall's contention that when the unpalatability is equal, the less numerous species will be attracted by the greater, but the greater will not be attracted by the less. Marshall does not fail to draw from this conclusion a very important consequence, for, as he points out, his premises lead to the inevitable conclusion that, when a mimetic similarity is once effected, the larger species will have gained the smaller share, but still $a$ share, of advantage from the association, and one might be inclined to argue from this that the larger species also will be led to approach this more advantageous condition. The far-reaching conclusion is drawn that such an argument is not valid, unless a continuous path from the first state to the second can be shown to exist, such that the advantage increases for each step along the path. Such a conclusion, if correct, would throw upon the selectionist an onus of detailed demonstration, which his opponent might increase indefinitely by challenging the details with increasing minuteness. Even if the case of Miillerian mimicry were not in itself of sufficient importance, it would be essential to examine in some detail the particular case in which the argument from ultimate advantage is believed to lead to an erroneous conclusion.

Marshall's argument is essentially as follows : if A and B are two equally distasteful species, of which $\mathrm{B}$ is the less numerous, then, in the absence of mistakes due to the resemblance of the two species, the young birds will take a proportionately heavier toll of $\mathrm{B}$ than of $\mathrm{A}$, before they have all learnt their lesson; consequently any mutant of A which resembles B will suffer more than the non-mutant type, and in consequence will be eliminated. It will be seen that the mutant is supposed to lose the whole of the advantage of the warning colour $\mathrm{A}$, and in return to receive only the less advantage of the warning colour $\mathrm{B}$, and this argument is indeed conclusive in showing that a mutation, which leaps clear outside the protective influence of its type, will suffer heavily for its rashness, even if, miraculously enough, its leap lands it in the heart of the protective influence of a less numerous aposeme. But what of a less violent mutation ? Is it possible to gain some of the advantage of resembling B without losing the whole of the advantage of resembling A? Is it even possible that increased shelter from aposeme B will more than counterbalance the loss from decreased shelter from aposeme A? In his answer to Marshall's argument Dixey (2) puts forward a directly opposite supposition, namely that a mutant of appearance intermediate between A and B, would gain the full advantage of both resemblances. In fact, whereas Marshall assumes that the whole of the advantage of resembling A is lost before any of the advantage of resembling B is gained, Dixey assumes, on the contrary, that the whole of the advantage of resembling B may be gained before any of the advantage of resembling A is lost. Both are clearly extreme assumptions; neither can be true generally, and since the two assumptions lead to opposite conclusions it would seem, as far as these arguments carry us, that we are faced with a balance of forces of unknown magnitude, and can neither assert that the Müllerian principle will work nor that it will fail.

There remains the argument upon which Muller relied, that the final condition of close resemblance being beneficial to both species, both will therefore tend to approach this advantageous condition. Marshall challenges the legiti- 
macy of this argument, his reason being the decisive one that he has disproved the conclusion in a particular instance; as we have seen, however, Marshall's argument in the chosen instance is indecisive, and the general argument from the advantage of the final state is in a position to reassert its claims. If it is true, however, it should be possible to devise a form of argument which shall show unequivocally, on the agreed postulates, that the admitted Miillerian situation will in fact produce a Müllerian evolutionary tendency affecting both species concerned,

Such an argument may, I suggest, be constructed by comparing the fate of any deviation from the type A, not with the average type, but with an equally conspicuous but opposite deviation. It will be admitted that variations of the species A, whether due to mutation or to Mendelian recombination, will be equally frequent in the direction of $\mathrm{B}$ as in the opposite direction; we may, therefore, without error, consider such variations to occur in pairs comprising variations of equal magnitude, but in opposite directions. Since they are of equal magnitude they will lose (if anything) equally by failing to be recognised as typically A, but if either, or both, are ever mistaken for species B, the greater benefit will certainly be reaped by the variation in the direction of B. Since the whole species may be regarded as made up of such pairs of variations, and since in every pair selection favours the one more like B, if either is favoured, the net resultant must be a modification in the direction of species B.

It will be seen that the condition for the existence of a mimetic tendency is that in a certain proportion of the situations in which $\mathrm{A}$ is liable to, but $\mathrm{B}$ is immune against attack, members of species A should, through their similarity to $\mathrm{B}$, actually escape attack. This is somewhat different from the condition arrived at by Prof. H. H. Turner, who speaks (7) of an actual overlap of the variations of the two species as the condition for the efficacy of Müller's statistical argument. The possibility of error on the part of the predator seems an essential feature in mimicry theory, and allowance can be made for it in Turner's treatment, provided we interpret his distribution curves as referring, not to the objective variability of the species, but to the (probably much greater) variability of the predator's subjective impressions, influenced as these must often be by inattention or haste, and by deceptive or insufficient illumination-in fact, by whatever circumstances conduce to error, human or avian. It is rather remarkable that, on a subject so remote from direct evidence as the subjective impressions of birds, we should possess three good reasons for assuming an approximately normal distribution : (a) that the reasons for which this distribution is chosen as the "normal law of errors" can scarcely be confined to mankind, (6) that the objective variability of a measurable character due either to Mendelian segregation, or to environmental fluctuations, is usually closely normal, and (c) that the resultant compounded of two independent distributions is nec essarily more normal than one, and possibly than both of its components.

The argument above developed may assuredly be refuted by disproving any of the biological factors assumed in the discussion. If it were proved that situations never in fact arise in which a member of A would survive if mistaken for B, but would perish if not so mistaken, that no predator learns by experience or is ever influenced by mimetic resemblances, or that such variations of $A$ as do favour the resemblance are not heritable, then the Müllerian theory of nunnery would fail as an explanation of the resemblances observed. The sole point established by the above reasoning is that if these biological factors are admitted the resulting evolutionary tendency cannot be confined to the less numerous of two species The efficiency of Müllerian selection will doubtless be greater 
(ceteris paribus) with the smaller species, but the supposed statistical objection to the Millerian attraction of a larger species (or group) by a smaller is wholly fictitious.

\section{THE THEORY OP SALTATIONS.}

Punnett (3, Chap. VI, pp. 72-74) repeats Marshall's argument, and concludes without reservation that Muillerian mimicry of a less numerous by a more numerous species is excluded by it. At first sight the argument appears irrelevant to Punnett's main contention of the inadequacy of Natural Selection to produce adaptations, for he evidently, unlike Marshall, would reject also both Batesian mimicry, and the Millerian mimicry, of the more numerous by the less numerous species. Nevertheless, it would not be altogether fair to regard Punnett's citation of Marshall's argument as a merely extraneous addition to his indictment, such as by arousing suspicion of error, though on an irrelevant issue, might serve to secure a verdict on the main count; on the contrary Marshall's argument plays a small yet essential part in his destructive argument derived from mimicry rings. The case of two presumably palatable female types each quite unlike the corresponding males, which males are unlike each other is chosen to illustrate this difficulty. The two females show an apparent mimetic resemblance to three other butterflies, two regarded as definitely unpalatable and the third as doubtfully so. Assuming that the non-mimetic males represent the former appearance of the two mimetic females, it is asked how the latter have come to resemble the distasteful members of the ring. Granted that these models might once have been not unlike in appearance to one of these males it can scarcely be assumed that they ever resembled both, either simultaneously or consecutively; but unless such a resemblance formerly existed a gradual mimetic evolution is precluded, and we should be forced to admit that the mimetic females arose as sports or saltations totally unlike their mothers. (The word "saltation" is used here in preference to "mutation" since the modern Mendelian usage of the latter word contains no suggestion of a pronounced difference. Mutations may be changes quite small compared to the individual variability of the species.)

It will be seen that for Punnett's argument on this important point, the gradual and mutual convergence of two or more different warning colours must be wholly excluded, for if the possibility of such a process is admitted the difficulty of imagining a continuous sequence of changes entirely disappears, while on the contrary the assumption of discontinuity becomes a burden upon the theory, involving as it does the definite improbability of hitting off a good resemblance at one shot. Consequently Marshall's argument, which Punnett seems to have taken as reimposing all the limitations of the Batesian situation, plays an essential part in the argument in favour of saltations; so essential indeed that it seems impossible to repair the breach made by its removal.

The case for saltations as presented by Punnett was not entirely negative and destructive in character; it embodied one (then) recently discovered fact of considerable interest, namely that the differences between the three forms of the trimorphic female of Papilio polytes could be ascribed to two Mendelian factors, both limited in their obvious effects to the female sex, and one apparently necessary for the manifestation of the second.

This fact is of importance as indicating the mechanism by which a clear polymorphism is maintained; it shows that polymorphism in this case, and probably in similar cases, is dependent on one or more Mendelian factors the function of which is to switch on one or other of the possible alternatives, just 
as the more widespread dimorphism of sex is also dependent upon the Mendelian mechanism. In some groups, e.g. Drosophila and Man, a whole chromosome is utilised in the process of sex determination, in some fishes, on the contrary, (6), crossing over has been found to occur between the "sex-chromosomes" in the male (the heterogametic sex in this group), and we evidently ought more properly to speak of the sex gene rather than the sex chromosome as the agent of sex determination. The passage from the one condition to the other, by the cessation of crossing over, presents no inherent difficulties, especially as Mendelian factors are known which expedite or inhibit crossing over. The reason for such a change is not so obvious, but since both systems are found still in use, it is probable that each has, upon particular conditions, its own advantages.

The core of Punnett's argument in favour of the production of mimetic forms by saltations lies in the Mendelian behaviour of the polymorphic females, for it is argued that these Mendelian factors must have arisen originally as mutations, and seeing that the different forms demonstrably differ by only single factor differences, these types must have sprung into existence each at a single leap. Convincing as this argument at first sight seems, we should, nevertheless, at once recognise our folly if we argued that because the sex difference in Lebistes is apparently determined by a single factor, therefore a female fish of that genus, with the appropriate adaptations of her sex had arisen by a single saltation from a male of the same species! Or vice versa. In this case we are freed even from the necessity of rejecting the supposed saltation as improbable, for since the reproduction of the species requires the co-operation of both sexes, we may be certain that the origin of the sex factor antedated the evolution of separate sexes, and has persisted, in its function of switch, unchanged during the whole course of the evolutionary development of these two types.

The example of sex emphasises strongly the fact that it is the function of a Mendelian factor to decide between two (or more) alternatives, but that these alternatives may each be modified in the course of evolutionary development, so that the morphological contrast determined by the factor at a late stage may be quite unlike that which it determined at its first appearance. The inference, therefore, that because a single factor determines the difference between a mimetic and a male-like form in $P$. polytes, therefore the mimetic form arose fully developed by a single mutation, is one that cannot fairly be drawn; it requires, in fact, the gratuitous assumption that no evolutionary change has taken place in the two alternative forms since the dimorphism was first established.

Certain genetical experiments have demonstrated that genetic changes of the kind here considered are compatible with a purely Mendelian scheme of inheritance. In rats, the hooded (black and white) pattern is a simple recessive to the "self" or "solid" coloration; the case is probably parallel to the "Dutch" pattern in rabbits, and the "recessive pied" in mice. In studying variations in the hooded pattern Castle (4) found that by selection it was easy to obtain strains of hooded rats which were almost entirely black, and other strains almost entirely white, and equally, of course, a large number of stable patterns of an intermediate character. All these types of hooded behaved as before, as simply recessive to self-colour. Two possible explanations were put forward; the first possibility was that the modification produced by selection lay in the hooded gene, that, in fact, selection had sorted out from a large number of slightly differing allelomorphs, those favouring much or little pigmentation, and consequently that the surviving hooded genes had been modified by selec- 
tion; the second possibility was that the hooded gene was invariable in character, but that the pigmented area depended also on the co-operation of other genes, so-called modifying factors, and that the change in the hooded pattern was the result of selection, among the alternatives presented by these modifiers, of those types which developed larger or smaller pigmented areas respectively, A crucial experiment was devised to decide between these possibilities. Rats of both selected lines were bred back to unselected selfs, the young were inbred, and the hooded pattern was recovered in the grandchildren; if the modification had taken place in the hooded gene the recovered hooded rats would have received fully modified hooded genes, and must have been as dark as the hooded line from which they were obtained; but, if other factors were responsible, the hooded grandchildren would have received these equally from their self and from their hooded grandparents, and would consequently be less dark than the latter. The second alternative was proved to be correct, the modification being readily transmitted by self-rats which contained no hooded gene. The gene, then, may be taken to be uninfluenced by selection, but its external effect may be influenced, apparently to any extent, by means of the selection of modifying factors.

Unless the above analogies are wholly misleading, we should suppose that the factors $\mathrm{H}$ and $\mathrm{R}$ which Fryer (5) found to determine the differences between the polymorphic forms of $P$. polytes, each arose suddenly by a mutation, and that the new genes so produced have been entirely unmodified since their first appearances. On the other hand, we should see no reason whatever on genetic grounds to believe that the combination HHrr on its first appearance at all closely resembled the modern form polytes, or was an effective mimic of $P$. aristolochiae; nor that the combination HHRR resembled the modern form romulus, or was an effective mimic of P. hector. The gradual evolution of such mimetic resemblances is just what we should expect if the modifying factors, which always seem to be available in abundance, were subjected to the selection of birds or other predators.

\section{STABILITY OF THE GENE-RATIO.}

It should be emphasised that there is nothing in the above argument which helps to explain polymorphism itself. The phenomenon is sufficiently uncommon to suggest that it must always owe its origin to some rather special circumstances; however, the Mendelian character of the phenomenon does suggest one short step in the direction of a solution, namely, that the underlying condition for its development is that the proportionate numbers of the genes of some Mendelian factor, having a fairly marked effect, should be in stable equilibrium. By stable equilibrium I wish to imply that, if this ratio is dis turbed from its equilibrium value it will automatically tend to return to that value, in whichever direction the disturbance takes place. Both Fryer and Punnett speak of the proportion of the Mendelian types being in stable equilibrium, in the absence of selection, and for particular values of the frequency ratio of the genotypes. Thus it seems to have been believed that the ratio is particularly stable if 44 per cent, are recessive, and this is compared with the observed percentage, 45 , as an indication that the species is in a stable state. It should be stated emphatically, firstly, that in the absence of selection the equilibrium of the gene-ratio is neutral, and is not properly described as stable, for on disturbance there will be no tendency to recover its former value; and, secondly, that all possible ratios either for the genes, or for the genotypes are 
on exactly the same level, no ratios being specially favoured. The only point to which, in this system, the term stability can properly be applied, is the ratio of the number heterozygous to the geometric mean of the numbers homozygous, and of this ratio there is no experimental evidence available; barring restrictions on random mating of a most drastic character, this ratio can scarcely differ much from its stable value, two.

That a genuine stability probably characterises the gene-ratio in polymorphic species appears probable from the consideration of the time required for a single mutation to increase sufficiently in numbers till it affects, say, onethird of the genes of the species. In the absence of selective advantage this time will be that of a number of generations of the same order as the number of individuals which there are in the species; for many species this would be at least a thousand times as long as the longest time allowable. In order to affect a considerable portion of the population within a reasonable time the new gene must enjoy a selective advantage. If this advantage were to continue, however large a proportion of the population were occupied by the favoured gene, the process would be continued until the extermination of its allelomorph, and no dimorphism would remain. Only if the selective advantage wanes with increasing numbers, disappears at a fixed ratio, and beyond that is reversed, will a true stability of the gene-ratio be established. Stability is thus not only evidence of selection, but of the dependence of selective advantage upon the actual ratio of the alternative types.

Stabilising selection can scarcely be other than exceptional, yet it may be expected to arise in several ways. A Batesian mimic, for example, will receive less protection, the more numerous it is in comparison with its model; a dimorphic Batesian mimic will therefore adjust the numbers of its two forms, if these are dependent upon a single Mendelian factor, until they receive equal protection; any increase in the numbers of one form at the expense of the other would diminish the advantage of the former and increase that of the latter, thus producing a selective action tending to restore the original proportion. Note that a mimic owing its advantage to Müllerian situations only should not be dimorphic unless additional causes of stability are at work, for apart from these the selection produces an unstable equilibrium, from which the ratio will continue to depart until one or other type is exterminated.

A second form of stabilising action is found in reproductive selection. The stable ratio of the sexes is clearly due to this cause, as is that of the thrum-eyed and pin-eyed primroses. It is interesting to note that Fryer, in his breeding experiments with Papilio polyles observed numerous cases of sterile unions, which suggested to him the possible existence of "illegitimate" pairings. One of the simplest possibilities of this type is a merely greater fertility of the heterozygous as compared to the homozygous condition. As I have shown elsewhere, (8), the stability in the gene-ratio of factors for which the heterozygote is favoured, affords also a possible explanation of the phenomenon of "hybrid vigour."

It should perhaps be noted that Gerould's work (9), on the dominant white observed in the female of several species of Colias, also reveals some peculiar features suggestive of a stability mechanism governing the yellow-white generatio. Gerould reports that great difficulties were encountered in obtaining the homozygous white types, these difficulties being evidently connected with the occurrence of a closely linked lethal factor. When pure white broods had been obtained, from a strain freed from the lethal, the failure of the males to mate caused the introduction of wild males, and these were found to bring in the 
lethal factor. The fact that this particular lethal is not apparently rare in nature, although we should expect it to die out somewhat rapidly, suggests that a stabilising system must be present. The genetic complexities are not fully elucidated, for certain types of mating seem regularly to give an abnormal sex-ratio (3 ?:2 ?). It is interesting in connection with the modifying effects of selection, that Gerould notes the occurrence of a fluctuating tinge of yellow on the wing of the genetically white female, and ascribes its variability to secondary factors.

Cases such as those of $\mathrm{P}$. polytes and Colias have an additional special interest, in that the stability mechanism probably involves both the reproductive elements of genetic lethality and sterility on the one hand, and on the other hand, elements involved in the differential adaptation, of the forms concerned, to survival in their wild environments; for if these latter were absent we should expect the gene-ratio to be the same in all localities. The unique possibility therefore presents itself of obtaining a direct measure of the selective value of such differential adaptation by equating it to the calculable effects of the reproductive selections, against which we find it in nature balanced. The importance of such a direct determination need scarcely be emphasised; the hindrances to free reproduction in these groups have appeared hitherto merely as an obstacle impeding the Mendelian analysis of the polymorphic forms; it is much to be hoped that, in view of the application outlined above, their elucidation may, in future studies, be made a principal object of research.

Whatever be the cause to which a factor owes its stability, any species in which a stable factor occurs will be potentionally dimorphic, and permanently so unless in changed conditions the stability can be upset. If, in this condition, selection favours different modifications of the two genotypes, it may become adaptively dimorphic by the cumulative selection of modifying factors, without alteration of the single-factor mechanism by which the dimorphism is maintained.

\section{SUMMARY.}

1. The contention of Marshall that statistical reasons preclude the action of selection from favouring the modification of a more numerous species in the direction of a closer resemblance to a similar but le ss numerous species, is without valid foundation.

2. The contention of Punnett that mimicry rings containing more than one palatable mimic, much modified from its primitive appearance, must have arisen by discontinuous saltation depends wholly on the validity of Marshall's argument.

3. The Mendelian behaviour of the different forms of a polymorphic species does not prove that these forms arose by single saltations.

4. The stability of the gene-ratios of factors controlling polymorphism implies a selective action, reproductive or other, influenced by the frequency-ratio of different forms. Any factor causing visible differences, and possessing a ratio of stable equilibrium, will provide a potential dimorphism capable of evolutionary development by the selection of modifying factors.

\section{REFERENCES.}

(1) MARSHALL, G. A. K. (1908). On diaposematism, with reference to some limitations of the Müllerian hypothesis of mimicry. Trans. Ent. Soc., 1908, pp. 93-142. 
(2) DIXEY, F. A. (1908). On Müllerian mimicry and diaposematism. Trans. Ent. Soc., 1908, pp. 559-583.

(3) PUnNeTt, R. C. (1915). Mimicry in butterflies. Camb. Univ. Press.

(4) CASTLE, W. E. (1914). Piebald rats and selection; an experimental test of the effectiveness of selection and of the theory of gametic purity in Mendelian crosses. Carnegie Inst. Publ., No. 195, pp. 56.

(5) FRYER, J. C. F. (1913). An investigation by pedigree breeding into the polymorphism of Papilio polytes. Phil. Trans., vol. 204, pp. 227-254.

(6) WINGE, O. (1923). Crossing over between the X-and Y-chromosome in Lebistes. Comptes Rendus des travaux du laboratoire de Carlsburg, vol. xiv, no. 20.

(7) TURNER, H. H. (1924). On the numerical as pect of reciprocal mimicry (Diaposematic resemblance). (Appendix to E. B. Poulton, Mimicry in the butterflies of Fiji, etc.). Trans. Ent. Soc., 1924, pp. 667-675.

(8) FISHER, R. A. (1922). On the dominance ratio. Proc. Roy. Soc. Edin., vol. xlii, pp. 321-341.

(9) GEROULD, J. H. (1923). Inheritance of white wing-colour, a sex-limited (sexcontrolled) variation in yellow Pierid butterflies. Genetics, vol. viii, pp. 495-551. 